\title{
Synchrotron Radiation from Ultra-High Energy Protons and the Fermi Observations of GRB 080916C
}

\author{
Soebur Razzaque ${ }^{1,2, *}$, Charles D. Dermer ${ }^{1, *}$ and Justin D. Finke ${ }^{1,2}$ \\ ${ }^{I}$ Space Science Division, U.S. Naval Research Laboratory, Washington, DC 20375, USA \\ ${ }^{2}$ National Academy of Sciences, Washington, DC 20001, USA
}

\begin{abstract}
Fermi $\gamma$-ray telescope data of GRB 080916C with $\sim 10^{55}$ erg in apparent isotropic $\gamma$-ray energy, show a several second delay between the rise of $100 \mathrm{MeV}-\mathrm{GeV}$ radiation compared with $\mathrm{keV}-\mathrm{MeV}$ radiation. Here we show that synchrotron radiation from cosmic ray protons accelerated in GRBs, delayed by the proton synchrotron cooling timescale in a jet of magnetically-dominated shocked plasma moving at highly relativistic speeds with bulk Lorentz factor $\Gamma \sim 500$, could explain this result. A second generation electron synchrotron component from attenuated proton synchrotron radiation makes enhanced soft X-ray to $\mathrm{MeV} \gamma$-ray emission. Long GRBs with narrow, energetic jets accelerating particles to ultra-high energies could explain the Auger observations of UHE cosmic rays from sources within $100 \mathrm{Mpc}$ for nano-Gauss intergalactic magnetic fields. The total energy requirements in a proton synchrotron model are $\propto \Gamma^{16 / 3}$. This model for GRB 080916C is only plausible if $\Gamma \leq 500$ and the jet opening angle is $\sim 1^{\circ}$.
\end{abstract}

Key Words: Gamma rays, bursts-gamma rays, theory-radiation mechanisms, nonthermal.

\section{INTRODUCTION}

An integrated fluence of $2.4 \times 10^{-4} \mathrm{erg} \mathrm{cm}^{-2}$ was measured from GRB 080916C with the Large Area Telescope (LAT) and Gamma ray Burst Monitor (GBM) on the Fermi Gamma ray Space Telescope, with one third of this energy in the LAT [1]. At a redshift $z=4.35 \pm 0.15$ [2], GRB 080916C has the largest apparent energy release yet observed from a GRB. A significant $\sim 4.5$ s delay between the onset of $>100 \mathrm{MeV}$ compared to the $\sim 8 \mathrm{keV}-5 \mathrm{MeV}$ radiation is found (the characteristic duration of the GBM emission is $\approx 50 \mathrm{~s}$ ).

The spectrum of GRB $080916 \mathrm{C}$ was fit by the smoothly connected double power-law Band function [3] to multi-GeV energies, though with changing Band spectral parameters and peak photon energy in different time intervals. The emergence of delayed spectral hardening is represented by a Band beta spectral index changing from $\beta=-2.6$ in the first 3.6 seconds following the GRB trigger to $\beta=-2.2$ at later times [1]. Here we show that a hard spectral component arising from cosmic-ray proton synchrotron radiation explains the delayed onset of the LAT emission. If GRBs accelerate UHECRs, then the delayed onset of the LAT emission after the GBM trigger should be a regular feature of GRB spectral evolution.

\section{PROTON ACCELERATION AND RADIATION}

GRB blast wave calculations usually treat electrons [4, 5], but protons and ions will also be accelerated if they are

*Address correspondence to these authors at the Space Science Division, U.S. Naval Research Laboratory, Washington, DC 20375, USA; Tel: (202) 404+1455; Fax: (202) 767-0497; E-mails: srazzaque@ssd5.nrl.navy.mil, charles.dermer@nrl.navy.mil present in the relativistic flows in black-hole jet systems. Here we consider protons accelerated in GRB blast waves to such energies that they can efficiently radiate hard $\sim \mathrm{GeV}-$ $\mathrm{TeV}$ photons by the proton synchrotron mechanism [6-11]. The highest energy photons are reprocessed by $\gamma \gamma \rightarrow e^{+} e^{-}$ opacity [12] to make an injection source of electrons and positrons that cool by emitting $<\mathrm{GeV}$ electron synchrotron radiation. Two delays arise, the first from the time it takes to accelerate protons to a saturation Lorentz factor where the acceleration rate equals the synchrotron rate. A second delay arises during which sufficient time passes to build up the spectrum of the primary protons so that they become radiatively efficient in the LAT band. Observations of GRB 080916C are consistent with the delayed onset between GBM and LAT emission being caused by the second delay where the evolving proton cooling synchrotron spectrum sweeps from higher energies into the LAT waveband. This radiation is emitted from a jet of magnetically-dominated shocked plasma with $\zeta_{B}>1$, where $\zeta_{B}$ is the ratio of magnetic-field to proton/particle energy density in the plasma.

The rapid variability, large apparent luminosity, and detection of high-energy photons from GRBs can be understood if this radiation is emitted from jetted plasma moving with bulk Lorentz factor $\Gamma>>1$ towards us. Detection of $3 \mathrm{GeV}$ and $13 \mathrm{GeV}$ photons from GRB 080916C suggests $\Gamma_{3}=\Gamma / 1000 \sim 1$ (Ref. [1] and below, Section 3). For variability times $t_{v} \sim 1 \mathrm{~s}$ and instantaneous energy fluxes $\Phi=10^{-5} \Phi_{-5}$ erg $\mathrm{cm}^{-2} \mathrm{~s}^{-1}$, the internal radiation energy density in the fluid is $u_{\gamma}^{\prime} \approx 4 \pi d_{L}^{2} \Phi /\left(4 \pi R^{2} c \Gamma^{2}\right) \approx(1+z)^{2} d_{L}^{2} \Phi /\left(\Gamma^{6} c^{3} t_{v}^{2}\right)$, implying a characteristic jet magnetic field of 


$$
B^{\prime}(\mathrm{k} G) \approx 2 \frac{\sqrt{\zeta_{B} \rho_{b} \Phi_{-5}}}{\Gamma_{3}^{3} t_{v}(\mathrm{~s})} \approx 2 \varsigma_{B}^{1 / 2} \rho_{b}^{1 / 2} t_{v}^{-1 / 2}(\mathrm{~s}), E_{1}^{-1 / 2}(13 \mathrm{GeV})
$$

where primes refer to the comoving frame, $\rho_{b}$ is the baryonloading parameter giving the relative energy in nonthermal protons compared to $\gamma$-rays. The last relation in equation (1) assumes $\Gamma \approx \Gamma_{\min }$ from the opacity condition $\tau_{r \gamma}=1$, which can be written as

$$
\Gamma_{\text {min }} \cong\left[\frac{\sigma_{\mathrm{T}} d_{L}^{2}(1+z)^{2} f_{\hat{\varepsilon}} \varepsilon_{1}}{4 t_{v} m_{e} c^{4}}\right]^{1 / 6},
$$

where $f_{\hat{\varepsilon}}$ is the $v F_{v}$ flux at photon energy $\hat{\varepsilon}=2 \Gamma^{2} /\left[(1+z)^{2} \varepsilon_{1}\right]$ (in $m_{e} c^{2}$ units) and $E=m_{e} c^{2} \varepsilon_{1}$ is the highest energy photon. Thus the total jet energy is $\propto \zeta_{B} \rho_{b} \Phi_{\gamma}$. A characteristic field $B^{\prime} \sim 10-100 \mathrm{kG}$ is consistent with the absence of a distinct self-Compton synchrotron component in GRB 080916C [1], which happens when the magnetic energy density is larger than the nonthermal electron energy density, or $\zeta_{B} \rho_{b}>1$.

In Fermi acceleration scenarios, protons gain energy on timescales exceeding the Larmor timescale, implying an acceleration rate $\dot{\gamma}_{a c c, p}^{\prime}=\phi^{-1} e B^{\prime} / m_{p} c$, where $\phi^{-1}<<1$ is the acceleration efficiency. Equating the acceleration rate with the synchrotron loss rate gives a saturation Lorentz factor for protons, namely

$\gamma_{s a t, p}^{\prime}=\frac{m_{p}}{m_{e}}\left(\frac{B_{c r}}{\phi B^{\prime}}\right)^{1 / 2} \sqrt{\frac{9}{4 \alpha_{f}}} \approx \frac{2 \times 10^{8}}{\sqrt{(\phi / 10) B_{5}^{\prime}}}$,

where $\alpha_{f}=1 / 137$ is the fine structure constant, $B_{c r}=m_{e}^{2} c^{3} / e \hbar=4.4 \times 10^{13} \mathrm{G}$ is the critical magnetic field, and the mean magnetic field of the radiating region is $B^{\prime}=10^{5} B_{5}^{\prime} \mathrm{G}$. The observer measures a time

$t_{\text {sat }}=\frac{1+z}{\Gamma} \frac{m_{p}^{2} c}{m_{e}} \sqrt{\frac{6 \pi \phi}{e \sigma_{T} B^{3}}} \approx \frac{0.01 \sqrt{\phi / 10}}{\Gamma_{3} B_{5}^{3 / 2}} \mathrm{~s}$

for protons to reach $\gamma_{s a t, p}^{\prime}$.

The proton synchrotron saturation frequency (in $m_{e} c^{2}$ units), corresponding to the proton synchrotron frequency of protons with $\gamma_{p}^{\prime}=\gamma_{s a t, p}^{\prime}$, is

$$
\varepsilon_{\text {sat }, p}=\frac{\Gamma}{1+z} \varepsilon_{\text {sat }, p}^{\prime}=\frac{\Gamma / \phi}{1+z} \frac{m_{p}}{m_{e}} \frac{27}{8 \alpha_{f}} \approx 1.6 \times 10^{7} \frac{\Gamma_{3}}{\phi / 10} .
$$

This frequency corresponds to a photon energy of $\approx 8$ $\mathrm{TeV}$ for GRB 080916C. Analogous to electron blast wave physics [13], proton synchrotron losses make a cooling break at the proton cooling Lorentz factor $\gamma_{c, p}^{\prime}=\gamma_{s a t, p}^{\prime}\left(t_{s a t} / t\right)$, which is obtained by equating the synchrotron energy-loss timescale with the comoving time $t^{\prime}=\Gamma t /(1+z)$. Consequently the proton synchrotron cooling frequency for protons with $\gamma_{p}^{\prime} \approx \gamma_{c, p}^{\prime}$ is $\varepsilon_{c, p}(t)=\varepsilon_{\text {sat }, p}\left(t_{s a t} / t\right)^{2}$ for our simplified model of continuous acceleration and uniform injection of particles during the first $\sim 8 \mathrm{~s}$ to the observer.
Photons with energies above $\sim 1-10 \mathrm{GeV}$ are strongly attenuated through $\gamma \gamma \rightarrow e^{+} e^{-}$processes in the source, inducing a nonthermal $e^{ \pm}$injection that makes a secondgeneration electron synchrotron component. Proton synchrotron photons radiated by protons with $\gamma_{p}^{\prime}=\gamma_{\text {sat }, p}^{\prime}$ materialize into electrons and positrons with Lorentz factor $\gamma_{e}^{\prime} \approx \varepsilon_{\text {sat }, p}^{\prime} / 2$. The electron synchrotron saturation frequency from this second generation of synchrotron emission is observed at

$\varepsilon_{\text {sat }, e} \approx \frac{3}{2} \frac{\Gamma}{1+z} \phi^{-2} \frac{B^{\prime}}{B_{c r}}\left(\frac{m_{p}}{m_{e}} \frac{27}{16 \alpha_{f}}\right)^{2} \approx \frac{10^{3} \Gamma_{3} B_{5}^{\prime}}{(\phi / 10)^{2}}$.

Therefore the second-generation synchrotron radiation cuts off above $\approx 600 \mathrm{MeV}$ for GRB 080916C. This will be below the LAT sensitivity because only the proton synchrotron radiation from protons with $\gamma_{p}^{\prime} \approx \gamma_{\text {sat }, p}^{\prime}$ contributes to the second-generation electrons and positrons that make emission at $\varepsilon \approx \varepsilon_{\text {sat }, e}$ and the number of such protons is small for a steep injection proton spectrum.

The direct proton synchrotron radiation from cooling protons equals the electron synchrotron saturation frequency when $\varepsilon_{c, p}(t)=\varepsilon_{\text {sat }, p}\left(t_{\text {sat }} / t\right)^{2}=\varepsilon_{\text {sat }, e}$, which is observed to take place at time

$t_{c l}=t_{s a t} \sqrt{\phi \frac{B_{c r}}{B^{\prime}} \frac{m_{e}}{m_{p}} \frac{64 \alpha_{f}}{81}}=\frac{4}{3} \frac{1+z}{\Gamma} \phi \frac{m_{p} c B_{c r}}{e B^{2}} \sqrt{\frac{m_{p}}{m_{e}}}$.

For GRB $080916 \mathrm{C}, \quad t_{c l} \approx 1.4(\phi / 10) / \Gamma_{3}^{-1} B_{5}^{\prime-2} \quad \mathrm{~s}$, which corresponds (depending precisely on $\varepsilon_{s a t, e}$ ) to the time required for proton synchrotron radiation to become strong in the LAT energy band. We propose this effect as the reason for the delayed onset of the LAT emission.

In addition to the direct proton synchrotron radiation, pair synchrotron radiation is formed from the internal attenuation of proton synchrotron photons to make ultra-relativistic pairs. If protons are accelerated with a number index $k$ (i.e., $\dot{N}(\gamma) \propto \gamma^{-k}$ ), then the cooling spectrum breaks from a proton distribution with index $k$ to a steeper one with index $k+1$. The proton synchrotron $v F_{v}$ flux, in the absence of $\gamma \gamma$ opacity, is simply

$$
\frac{f_{\varepsilon}^{p, s y n}}{f_{\varepsilon_{s a t, p}, p s n}}=\left\{\begin{array}{l}
\left(\frac{\varepsilon_{c, p}}{\varepsilon_{s a t, p}}\right)^{\frac{2-k}{2}}\left(\frac{\varepsilon_{\text {min }, p}}{\varepsilon_{c, p}}\right)^{\frac{3-k}{2}}\left(\frac{\varepsilon}{\varepsilon_{\text {min }, p}}\right)^{\frac{4}{3}}, \varepsilon<\varepsilon_{\text {min }, p} \\
\left(\frac{\varepsilon_{c, p}}{\varepsilon_{s a t, p}}\right)^{\frac{2-k}{2}}\left(\frac{\varepsilon}{\varepsilon_{c, p}}\right)^{\frac{3-k}{2}}, \varepsilon_{\text {min }, p}<\varepsilon<\varepsilon_{c, p}(t) \\
\left(\frac{\varepsilon}{\varepsilon_{s a t, p}}\right)^{\frac{2-k}{2}}, \varepsilon_{c, p}(t)<\varepsilon<\varepsilon_{s a t, p}
\end{array}\right.
$$


where $f_{\varepsilon_{s a t, p}}^{p, s y n}=\Psi \rho_{b} \Phi_{\mathrm{G} B M}, \gamma_{m i n, p}^{\prime} \approx \Gamma_{r e l}$, the relative Lorentz factor of the relativistic wind in the jet and the shell material, and

$\Psi=\left(\frac{k-2}{2}\right) \frac{1-\left(\varepsilon_{m i n, p} / \varepsilon_{s a t, p}\right)^{(3-k) / 2}}{\left(\varepsilon_{m i n, p} / \varepsilon_{s a t, p}\right)^{(2-k) / 2}-1} ;$

$\Psi=\left[\ln \left(\gamma_{\text {sat }, p}^{\prime} / \gamma_{\min , p}^{\prime}\right)\right]^{-1}$ when $k=2$.

The direct proton synchrotron flux from GRB 080916C is attenuated at all energies from $\varepsilon_{\gamma /} \approx 1-10 \mathrm{GeV}$ to $\varepsilon \approx \varepsilon_{\text {sat, } p}$, equation (5), to make a second-generation electron injection spectrum with the same form as equation (8), though with subscript $p \rightarrow e$ spectral indices $(3-k) / 2 \rightarrow(3-k) / 4$ and $(2-k) / 2 \rightarrow(2-k) / 4$ and $f_{\varepsilon_{s a t, e}}^{e, s y n}=\frac{1}{2} f_{\varepsilon_{s a t, p}}^{p, s y n}$. The second-generation spectrum has a lowenergy cut-off related to $\varepsilon_{\gamma \gamma}$, below which it receives no further injection pairs. Note that $\varepsilon_{c, e}(t)=\varepsilon_{\text {sat }, e}\left(t_{s a t} / t\right)^{4}$, where $\varepsilon_{\text {sat }, e}$ is given by equation (6).

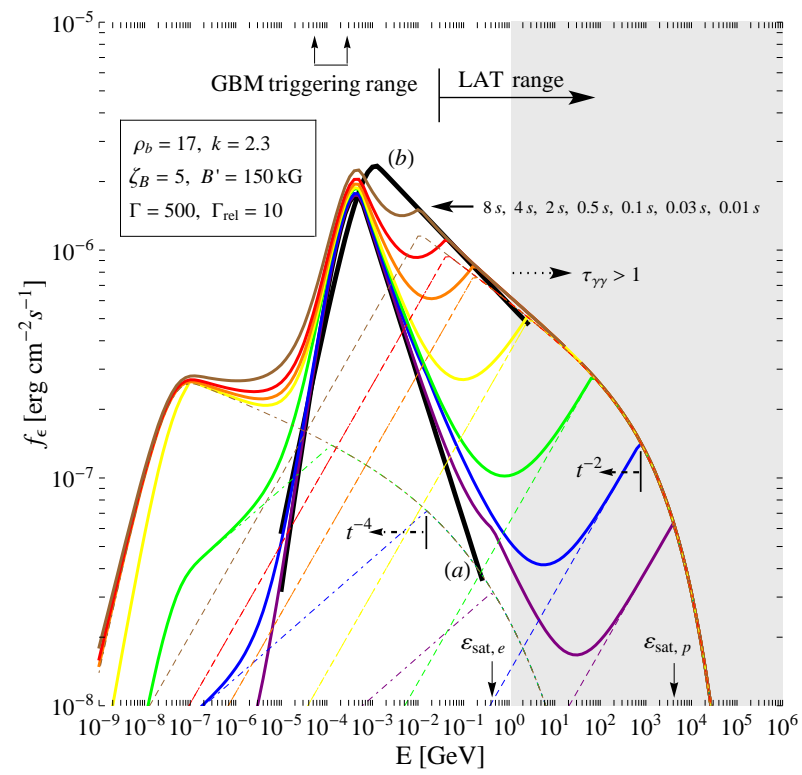

Fig. (1). Synchrotron model for the $\gamma$-ray spectrum of GRB 080916C. The $v F_{v}$ flux in the GBM range is produced by primary nonthermal electron synchrotron radiation. The heavy dark curves show the fitted Band spectrum for intervals (a), from 0 to $3.6 \mathrm{~s}$ after the trigger, and interval (b), from $3.6 \mathrm{~s}$ to $7.7 \mathrm{~s}$. A strong proton synchrotron component is formed at high energies and sweeps into the LAT band after several seconds, thus making the time delay between the GBM and LAT emissions. A weak pair synchrotron $\gamma$ ray component is formed in the GBM and LAT band from secondgeneration pair synchrotron radiation formed by proton synchrotron photons that are attenuated by $\gamma \gamma$ processes in the jet. Here we assume uniform injection over $8 \mathrm{~s}$, and take $\phi=10$. At a fixed photon energy below the cooling frequency, the $v F_{v}$ flux increases $\propto t$ for both the proton synchrotron and pair synchrotron radiation.
Fig. (1) shows the evolving proton synchrotron spectrum for constant injection with time using the above relations for parameters of GRB 080916C, with the Band function in interval (a), the first $3.6 \mathrm{~s}$ following the trigger. The Band function in the $\sim 10 \mathrm{keV}$ to $\leq 100 \mathrm{MeV}$ range can be due to synchrotron radiation by primary electrons, or thermal radiation from a jet photosphere. For the proton spectrum, we use $k=2.3, \rho_{b}=17, \zeta_{B}=5$, and $\gamma_{\text {min, } p}^{\prime}=10$. In this simple picture of the GRB jet, the protons are accelerated to the saturation Lorentz factor within $\approx 0.01 \mathrm{~s}$, making a prompt second-generation electron synchrotron spectrum too weak to be detected, followed after a few seconds by strong direct proton synchrotron emission that sweeps into the LAT band from high energies. The time delay in the high-energy $\gamma$-ray flux occurs even for variable injection, provided it occurs over $\sim 8 \mathrm{~s}$ to the observer. Although the data for GRB 080916C are consistent with a Band function, spectral analyses with the addition of a proton synchrotron component will determine whether this more complicated model is compatible with Fermi LAT GRB data.

Implicit in the treatment is that the wind impacts either a shell ejected earlier by the central engine or some roughly uniform density material that already existed in the surrounding medium. The extent of this material is $\langle r\rangle \approx c \Gamma^{2} \Delta t /(1+z) \approx 5 \times 10^{16} \Gamma_{3}^{2}(\Delta t / 8 \mathrm{~s}) \mathrm{cm}$. The magnetic field is thus assumed to be roughly constant over the first $\sim 10$ s because of the constant density medium that is being swept up at the shock. This assumption is supported by the roughly constant value of electron synchrotron energy flux $\Phi_{\mathrm{GBM}}$ measured with the GBM, in the same time interval [1].

\section{TOTAL ENERGY OF GRB 080916C}

The comoving synchrotron cooling timescale of an ion with atomic mass $A$ and charge $Z$ is given by $t_{\text {syn }}^{\prime}=\left(A^{3} / Z^{4}\right)\left(m_{p} / m_{e}\right)^{3}\left(6 \pi m_{e} c / \sigma_{\mathrm{T}} B^{2} \gamma\right)$. The comoving peak synchrotron photon energy is $\varepsilon_{s y n}^{\prime}=(Z / A)\left(m_{e} / m_{p}\right) B \gamma^{2} / B_{c r}$. Equating the observer time $t_{s y n}=(1+z) t_{s y n}^{\prime} / \Gamma$ for the emission to be radiated at measured energy $m_{e} c^{2} \varepsilon_{s y n}=\Gamma \varepsilon_{s y n}^{\prime} /(1+z)=100 E_{100} \quad \mathrm{MeV}$ implies a comoving magnetic field $B^{\prime}(\mathrm{G}) \cong 2.0 \times 10^{5} A^{5 / 3} Z^{-7 / 3} E_{100}^{-1 / 3} t_{s y n}^{-2 / 3}$ (s) and an isotropic jet power, dominated by magnetic-field energy, given by

$$
L_{B} \cong \frac{R^{2} c \Gamma^{2} B^{\prime 2}}{2} \cong 2 \times 10^{58} \frac{\Gamma_{3}^{16 / 3} A^{10 / 3} t_{s y n}^{2 / 3}(s)}{Z^{14 / 3} E_{100}^{2 / 3}} \mathrm{erg} \mathrm{s}^{-1}
$$

letting the blast-wave radius $R \cong \Gamma^{2} c t /(1+z)$. For $\mathrm{Fe}$ ( $A=56, Z=26$ ), the power requirements are reduced by a factor $\approx 0.17$. Here, however, we consider only proton synchrotron radiation.

Eq. (10) shows that $L_{B} \propto \Gamma^{16 / 3}$ [11]. The absolute jet power varies as the square of jet opening angle $\theta_{j}$. In Ref. [1], only the uncertainty in the redshift was used to provide 
uncertainty in $\Gamma_{\min }$ using simple $\gamma \gamma$ opacity arguments, Furthermore, validity of the cospatial assumption that the soft photons are found in the same region as the hard photons was assumed in the calculation of $\Gamma_{\min }$. We use a likelihood ratio test to calculate the chance probability to detect a photon with energy greater than the maximum measured photon energy in a given time bin. For a -2.2 photon number spectrum, this test gives values of $\Gamma=(0.90,0.82,0.75) \Gamma_{\min }$ for exponential escape and $\Gamma=(0.79,0.58,0.36) \Gamma_{\min }$ for slab or spherical escape at the $(1 \sigma, 2 \sigma, 3 \sigma)$ levels, respectively.

If Type Ib,c supernovae are the progenitors of long GRBs like GRB 080916C, then $\theta_{j} \geq 0.8^{\circ} \quad$ [14]. Taking a conservative lower limit $\Gamma_{3} \cong 0.5$ gives the absolute energy requirements for GRB 080916C of $E \cong 3 \times 10^{53}\left(\Gamma_{3} / 0.5\right)^{16 / 3}\left(\theta_{j} / 1 \mathrm{deg}\right)^{2}\left(t_{\text {syn }} / 8 \mathrm{~s}\right)^{5 / 3}$ erg after integrating eq. (10) over time and multiplying by a two-sided jet beaming factor $f_{b} \cong 1.5 \times 10^{-4}\left(\theta_{j} / 1 \mathrm{deg}\right)^{2}$. The rotational energy available in a core collapse supernova could be as large as $\approx 5 \times 10^{54}$ erg for a $10 M_{\text {sol }}$ core [15].

Although it appears to be a coincidence that $\Gamma \approx \Gamma_{\min }$ in our model, which is required because of excessive powers when $\Gamma \gtrsim \Gamma_{\min }$, more complicated geometries might relax the bulk Lorentz factor requirement further. If the inner engine makes the prompt $\mathrm{MeV}$ radiation and residual shell collisions at larger radii make LAT $\gamma$-ray photons, then $\Gamma$ could be as low as $\sim 300$ [16]. In this case, the absolute energy release could be as low as $\approx 2 \times 10^{52}\left(\theta_{j} / 1 \mathrm{deg}\right)^{2}$ erg. Even though the model in Ref. [16] provides a separate explanation for the delayed onset (and predicts that the variability timescale of the $>100 \mathrm{MeV}$ radiation is longer than the $\mathrm{keV} / \mathrm{MeV}$ radiation), lack of cospatiality does not guarantee delayed onset. For example, photospheric emission with leptonic emission from internal shells would neither be cospatial nor necessarily exhibit a delayed onset.

The jet break time with apparent isotropic energy release $\approx 2 \times 10^{57}$ ergs is $t_{b r} \cong 0.3\left(\theta_{j} / 1 \mathrm{deg}\right)^{16 / 3} n_{0}^{-1 / 3} \mathrm{~d}$. The jet break would have taken place before Swift slewed, at $\approx T_{0}+17.0$ hr, to GRB 080916C [17], with a hard electron spectrum to explain the shallow X-ray decay observed $\gtrsim 10^{5} \mathrm{~s}$ after the GRB [18].

$\mathrm{A} \approx 3 \mathrm{~s}$ and $\approx 2 \mathrm{~s}$ delayed onset of $\geq 100 \mathrm{MeV}$ emission detected in another long GRB 090902B [19] and in the short GRB 081024B [20], respectively, could be explained with proton synchrotron radiation as we discussed here, although lack of redshift measurement for GRB 081024B prohibits calculation of the bulk Lorentz factor and the total $\gamma$-ray energy. It is, however, interesting to note that Fermi-LATdetected short GRBs emit higher fluence in the LAT range than in the GBM range $[20,21]$ which could be due to hadronic emission processes.

\section{ULTRAHIGH-ENERGY COSMIC RAYS FROM GRBS}

GRBs have long been considered as candidate sources to accelerate UHECRs [22, 23]. The energy of protons with $\gamma_{p}^{\prime} \approx \gamma_{s a t, p}^{\prime}$, if they were to escape from the GRB blast wave, is $\approx 2 \times 10^{20} \Gamma_{3} / \sqrt{(\phi / 10) B_{5}^{\prime}} \mathrm{eV}$, so GRB 080916C can in principle accelerate UHECRs. The Larmor timescale $t_{e s c}^{\prime}(\mathrm{s}) \approx 0.2 / \sqrt{(\phi / 10) B_{5}^{\prime 3}}$ at $\gamma_{p}^{\prime} \approx \gamma_{\text {sat }, p}^{\prime}$ is much less than the light-crossing time $t_{l c}^{\prime}(\mathrm{s}) \approx 200 t_{v}(\mathrm{~s})$, so that escape depends on transport in the jet plasma magnetic field impedes escape [24]. Photohadronic processes can assist escape by converting protons to neutrons, but are more important when the internal photon density is high, or when the variability timescale $t_{v}$ is small [25]. When $t_{v}(\mathrm{~s})<0.01\left(\Phi_{\mathrm{G} B M} / 10^{-5} \mathrm{erg} \mathrm{cm}^{-2} \mathrm{~s}^{-1}\right) /\left(\Gamma_{3}^{4} \varepsilon_{p k}\right)$, then photopion losses are important in GRB 080916C (see Ref. [26] for applications to GRB 090510). The shortest variability timescale for GRB 080916C observed with INTEGRAL is $0.1 \mathrm{~s}$ [2], so unless the corresponding size scale for the radiating region was even shorter, we can neglect photohadronic processes. But a shorter variability timescale would require a larger bulk Lorentz factor $\Gamma$ in order to explain detection of multi-GeV photons, in which case photohadronic efficiency is reduced [10].

We estimate the rate of long-duration GRBs as energetic as GRB $080916 \mathrm{C}$ within the $\approx 100 \mathrm{Mpc}$ clustering radius for UHECRs observed with Auger [27, 28]. For maximum total energy releases of $\approx 10^{54} \mathrm{erg}$, the GRB $080916 \mathrm{C}$ jet opening angle $\theta_{j}<100 / \Gamma=0.1 / \Gamma_{3}$. The inferred GRB rate of $\approx 2 f_{b} \mathrm{Gpc}^{-3} \mathrm{yr}^{-1}$ [29] at the typical redshift $z=1-2$ is a factor $\approx 1 / 10$ smaller at $\approx 100 d_{100} \mathrm{Mpc}$ due to the star formation rate factor and a factor $f_{b}>200$ larger due to a beaming factor. A $60 E_{60} \mathrm{EeV}$ UHECR is deflected by an angle $\approx 4.4^{\circ} Z B_{n G} E_{60}^{-1} d_{100}^{1 / 2} \lambda_{1}^{1 / 2}$ in intergalactic magnetic field with mean strength $B_{n G} \mathrm{nG}$ and coherence length of $\lambda_{1}$ Mpc. Deflection causes dispersion in time of arrival of UHECRs [30] and increases the apparent rate. The corresponding number of GRB sources within $\approx 100 \mathrm{Mpc}$ with jets pointing within $4^{\circ}$ of our line-of-sight is $\approx 30\left(f_{b} / 200\right) B_{n G}^{2} E_{60}^{-2} \lambda_{1}^{3 / 2}$. Complications arising from nonuniform magnetic field geometries (e.g. Refs. [31, 32]) can lead to different values in the rate estimates, but still allow GRBs as UHECR hosts. Thus if typical long duration GRBs have a narrow, highly relativistic core accelerating UHECRs, then long-duration GRBs could account for the Auger events within the GZK radius.

\section{DISCUSSION}

We have developed a hadronic model based on synchrotron radiation by protons in a highly magnetized shocked plasma, to explain delayed onset of high energy ( $\geq$ $100 \mathrm{MeV}$ ) emission observed with Fermi from GRB 
080916C. This proton synchrotron spectral component, in addition to the electron synchrotron and photospheric emission [33] that dominates in the $\mathrm{keV}-\mathrm{MeV}$ range, initially starts at much higher energy and later sweeps into the Fermi LAT range, thus causing a time delay in the prompt phase. Our model is compatible with an internal shocks scenario and could also be consistent with an origin of the high-energy emission in GRB 080916C from an external shock [34]. The late time extended emission observed in GRB 080916C and other GRBs could be due to the slower cooling of the protons in the early afterglow related to the external shock $[6,9]$.

Our approach differs from the conclusions of [35] that the jet outflow energy in GRB 080916C is dominated by Poynting flux rather than particle energy. Their conclusion depends on the assumption that the engine radius $r_{0} \approx c t_{v} /(1+z)$, whereas $r_{0}$ might be much smaller. In our scenario, both the relativistic outflow and the shell on which it impacts are particle energy dominated; only the shocked fluid is highly magnetized. The GBM emission could be nonthermal electron synchrotron radiation that includes photospheric emission made at smaller radii than the nonthermal synchrotron radiation. Compton cooling of shocked electrons is suppressed in highly magnetized shocked plasma, but could be important in a leptonic model with external cocoon photons [36]. Our model for GRB 080916C also applies to other Fermi LAT GRBs with comparable $\Gamma$ factors and small beaming angles.

Besides GRBs, UHECRs might also be accelerated in other systems of relativistic outflows, including low luminosity GRBs [37, 38], radio galaxies [39] and blazars. UHECRs could be formed through neutron escape when photopion processes are important, which will require IceCube neutrino detections [40] to establish. In GRB 080916C, where multi-GeV radiation is observed with Fermi, we have shown that synchrotron radiation from ultrahigh energy protons accelerated in GRB jets explains the delay of the $\geq 100 \mathrm{MeV}$ LAT emission with respect to the $\mathrm{keV}-\mathrm{MeV}$ GBM emission, and that long duration GRBs are possible sites of UHECR acceleration.

\section{ACKNOWLEDGEMENTS}

This work is dedicated to the memory of David L. Band.

We thank Armen Atoyan, Eduardo do Couto e Silva, Jonathan Granot, Francesco Longo, Peter Mészáros, Nicola Omodei, Kenji Toma, Xuefeng Wu, Ryo Yamazaki, and anonymous referees for comments. This work is supported by the Office of Naval Research and NASA.

\section{REFERENCES}

[1] Abdo AA, Ackermann M, Arimoto M, et al. Fermi Observations of High-Energy Gamma-Ray Emission from GRB 080916C. Science 2009; 323: 1688.

[2] Greiner J, Clemens C, Krühler T, et al. The redshift and afterglow of the extremely energetic gamma-ray burst GRB 080916C. Astron Astrophys 2009; 498: 89-94.

[3] Band D, Matteson J, Ford L, et al. BATSE observations of gammaray burst spectra. I - Spectral diversity. Astrophys J 1993; 413: 281-92.

[4] Piran T. The physics of gamma-ray bursts. Rev Mod Phys 2004; 76: $1143-210$

[5] Mészáros P. Gamma-ray bursts. Rep Prog Phys 2006; 69: 2259.
[6] Böttcher M, Dermer CD. High-energy Gamma Rays from Ultrahigh-energy Cosmic-Ray Protons in Gamma-Ray Bursts. Astrophys J Lett 1998; 499: L131.

[7] Totani T. Very strong tev emission as gamma-ray burst afterglows. Astrophys J Lett 1998; 502: L13.

[8] Totani T. TEV burst of gamma-ray bursts and ultra-high-energy cosmic rays. Astrophy J Lett 1998; 509: L81-L84.

[9] Zhang B, Mészáros P. High-Energy spectral components in gamma-ray burst afterglows. Astrophys J 2001; 559: 110-22.

[10] Razzaque S, Dermer CD, Finke JD, Atoyan A. Observational consequences of grbs as sources of ultra high energy cosmic rays. GAMMA-RAY BURST: Sixth Huntsville Symposium. AIP Conference Proceedings 2009; vol. 1133: pp. 328-333.

[11] Wang X-Y, Li Z, Dai Z-G, Mészáros P. GRB 080916C: On the Radiation Origin of the Prompt Emission from $\mathrm{keV} / \mathrm{MeV}$ TO GeV. Astrophys J Lett 2009; 698: L98-L102.

[12] Razzaque S, Mészáros P, Zhang B. GeV and higher energy photon interactions in gamma-ray burst fireballs and surroundings. Astrophys J 2004; 613: 1072-78.

[13] Sari R, Piran T, Narayan R. Spectra and light curves of gamma-ray burst afterglows. Astrophys J Lett 1998, 497: L17.

[14] Soderberg AM, Nakar E, Berger E, Kulkarni SR. Late-Time radio observations of 68 type ibc supernovae: strong constraints on offaxis gamma-ray bursts. Astrophys J 2006; 638: 930-37.

[15] Paczynski B. Are gamma-ray bursts in star-forming regions? Astrophys J Lett 1998; 494: L45.

[16] Li Z, Prompt $\mathrm{GeV}$ emission from residual collisions in GRB outflows: evidence from fermi observations of GRB 080916c. Astrophys J 2010; 709: 525-534.

[17] Hoversten EA, Schady P, Perri M. GRB 080916C: Swift UVOT refined analysis. GRB coordinates network, circular service 2008 ; http: //cdsadps:U-Strasbg-fr/abs/2008GCN.. 88262 ...1H.

[18] Stratta G, Perri M, Preger B, et al. Swift observation of the fermidetected GRB 080916C. GCN Report 166, 2008; pp. 1-2.

[19] Abdo AA, Ackermann M, Ajello M, et al. Fermi observations of GRB 090902B: a distinct spectral component in the prompt and delayed emission. Astrophys J Lett 2009, 706, L138-L144.

[20] Abdo AA, Ackermann M, Ajello M, et al. Fermi detection of delayed gev emission from the short gamma-ray burst 081024B. Astrophys J 2010, 712; 558-64.

[21] Le T, Dermer CD. Gamma-ray burst predictions for the fermi gamma ray space telescope. Astrophys J 2009; 700: 1026-33.

[22] Waxman E. Cosmological gamma-ray bursts and the highest energy cosmic rays. Phys Rev Lett 1995; 75: 386.

[23] Vietri M. The acceleration of ultra-high-energy cosmic rays in gamma-ray bursts. Astrophys J 1995; 453: 883.

[24] Dermer CD. Rapid x-ray declines and plateaus in swift grb light curves explained by a highly radiative blast wave. Astrophys J 2007; 664: 384.

[25] Dermer CD, Atoyan A. High-energy neutrinos from gamma ray bursts. Phys Rev Lett 2003; 91: 071102 .

[26] Asano K, Guiriec S, Mészáros P. Hadronic models for the extra spectral component in the short GRB 090510. Astrophys J Lett 2009; 705: L191.

[27] Abraham J, Abreu P, Aglietta, et al. Correlation of the highestenergy cosmic rays with nearby extragalactic objects. Science 2007; 318: 938-43

[28] Abraham J, Abreu P, Aglietta, et al. Astrophysical sources of cosmic rays and related measurements with the pierre auger observatory. submissions to the 31st International Cosmic Ray Conference, Lodz, Poland, July 2009. Available from: http://fr.arxiv.org/abs/0906.2347

[29] Guetta D, Piran T, Waxman E. The luminosity and angular distributions of long-duration gamma-ray bursts. Astrophys J 2005; 619: 412

[30] Waxman E, Coppi P. Delayed GeV-TeV photons from gamma-ray bursts producing high-energy cosmic rays. Astrophys J 1996; 464: L75.

[31] Kashti T, Waxman E. Searching for a correlation between cosmicray sources above $10^{19} \mathrm{eV}$ and large scale structure. JCAP 2008; 5: 6.

[32] Murase K, Zhang B, Takahashi K, Nagataki S. High-energy emission as a test of the prior emission model for gamma-ray burst afterglows. Mon Not R Astron Soc 2009; 396: 1825.

[33] Pe'er A, Ryde F, Wijers RAMJ, Mészáros P, Rees MJ. A new method of determining the initial size and lorentz factor of gamma- 
ray burst fireballs using a thermal emission component. Astrophys J Lett 2007; 664: L1.

[34] Kumar P, Barniol Duran R. On the generation of high-energy photons detected by the fermi satellite from gamma-ray bursts. Mon Not R Astron Soc 2009; 400: L75-L79.

[35] Zhang B, Pe'er A. Evidence of an initially magnetically dominated outflow in GRB 080916C. Astrophys J Lett 2009; 700: L65.

[36] Toma K, Wu X-F, Mészáros P. An up-scattered cocoon emission model of gamma-ray burst high-energy lags. Astrophys J 2009; 707: 404-16.
[37]

Murase K, Ioka K, Nagataki S, Nakamura T. High-Energy neutrinos and cosmic rays from low-luminosity gamma-ray bursts? Astrophys J Lett 2006; 651: L5.

[38] Wang X-Y, Razzaque S, Mészáros P, Dai Z-G. High-energy cosmic rays and neutrinos from semirelativistic hypernovae. Phys Rev D 2007; 76: 083009.

[39] Stanev T, Biermann PL, Lloyd-Evans J, Rachen JP, Watson AA. Arrival directions of the most energetic cosmic rays. Phys Rev Lett 1995; 75: 3056.

[40] Ahrens J, Bahcall JN, Bai X, et al. Sensitivity of the IceCube detector to astrophysical sources of high energy muon neutrinos. Astropart Phys 2004; 20: 507-32.

(C) Razzaque et al.; Licensee Bentham Open.

This is an open access article licensed under the terms of the Creative Commons Attribution Non-Commercial License (http://creativecommons.org/licenses/by-nc/3.0/) which permits unrestricted, non-commercial use, distribution and reproduction in any medium, provided the work is properly cited. 